\title{
Advanced Constitutive Modelling for Creep-Fatigue Assessment of High Temperature Components
}

\author{
Ehsan Hosseini ${ }^{1}$, Stuart Holdsworth ${ }^{2}$, Ingo Kühn ${ }^{3}$ and Edoardo Mazza ${ }^{4}$ \\ ${ }^{1}$ Inspire Centre for Mechanical Integrity, Switzerland \\ ${ }^{2}$ EMPA, Swiss Federal Laboratories for Materials Science \& Technology, Switzerland \\ ${ }^{3}$ ALSTOM Power Ltd, Baden, Switzerland \\ ${ }^{4}$ ETHZ, Swiss Federal Institute of Technology, Switzerland
}

\begin{abstract}
Creep-fatigue assessment procedures for the design of high temperature components such as rotating turbo-machinery parts should ensure lifetime predictions which are safe but not excessively conservative. Adoption of inherently safe, but more accurate and less conservative high temperature assessment procedures than are presently available enable the availability of power plant with greater operating flexibility. Operating flexibility is becoming a key market driver due to increased interest in the use of intermittent renewable energy sources (e.g. wind, solar, etc.) which place focus on a requirement for turbo-machinery to be capable of reduced start-up and shut-down times in order to compensate for undersupplies provided by these new technologies. This study introduces a creep-fatigue assessment procedure for the design of high temperature components required for flexible operation. In particular, it considers alloys with high creep-fatigue deformation/damage interaction characteristics such as the advanced martensitic $9-11 \% \mathrm{Cr}$ steels which are widely used for power plant applications. The procedure takes advantages of advanced constitutive models for both cyclic plasticity and creep and implements them in a state-of-the-art mechanical assessment procedure for calculating high temperature component life times. In the absence of appropriate service experience, the effectiveness of the adopted creep-fatigue assessment procedure for lifetime prediction is being evaluated using the results from a set of component feature specimen service-cycle thermomechanical fatigue benchmark tests.
\end{abstract}

\section{INTRODUCTION}

The growing awareness of the detrimental environment impact of fossil energy sources and the potential safety risks associated with nuclear energy are increasingly encouraging the employment of renewable energy resources. The intermittent character of renewable energy supplies (e.g. solar and wind) however present a key obstacle for their full substitution and the traditional power plants are still needed to balance energy generation when renewable energy production is down or insufficient. This has introduced the requirement for changes in the operating conditions of traditional (non-renewable) power plants. The start-up of the traditional power plants (e.g. base load power plants) was very slow and took many hours to achieve a steady-state power output. The plant then continued operating for considerably long duration before an interruption. The current market however demands for quicker and efficient start-up and shutdown cycles to accommodate the fluctuation of renewable energy sources. Such fast start-up and shutdown transients generate considerable temperature gradients, for example, within rotating turbomachinery parts and develop high thermal strains/stresses. In contract to traditional turbo-machinery where creep was the main damaging mechanism, the relatively high frequency (e.g. even more than once a day) of fast start-up transients required for current turbo-machinery are responsible for an increased induce of thermo-mechanical fatigue (TMF) damage and the creep-fatigue interaction. One of the main challenges in the design of components under such thermo-mechanical creep-fatigue condition is their lifetime prediction. 
This study aims to introduce a creep-fatigue assessment procedure for the design of high temperature components operating under thermo-mechanical creep-fatigue conditions (e.g. rotating turbo-machinery parts) which is inherently safe, but more accurate and less conservative than present procedures. In particular, it considers alloys with high creep-fatigue deformation/damage interaction characteristics such as the advanced martensitic $9-11 \% \mathrm{Cr}$ steels which are widely used for power plant applications. The procedure takes advantages of advanced constitutive models for both cyclic plasticity and creep, and implements them in a state-of-the-art mechanical assessment procedure for calculating high temperature component life times. At the same time, the complexity and amount of numerical efforts has been minimised to enable industrial application of the procedure.

The employed constitutive models for cyclic-plasticity and creep represent the material behaviour for mid-life cycle conditions and consider important phenomena such as primary creep persistency (PCP, Radosavljevic, 2011). The design analysis of real components cannot be based on the behaviour description of a single heat of an alloy and is typically based on mean alloy properties for the determination of creep-fatigue deformation, and minimum alloy properties for the determination of creepfatigue damage behaviour. The cyclic plasticity and creep constitutive models are therefore refined here to capture the heat-to-heat variability of the alloy deformation response and represent the mean alloy properties.

Several low cycle fatigue (LCF), cyclic/hold $(\mathrm{C} / \mathrm{H})$ and creep tests at different temperatures and for different heats of the alloy had been conducted to provide necessary information for employment of the introduced creep-fatigue assessment procedure for component/structure assessment (Hosseini et al., 2015a). One solution, in the absence of appropriate service experience, is to verify the effectiveness of new assessment procedures applied to components manufactured from new materials and operated in new ways using the results from component feature specimen service-cycle TMF benchmark tests (Holdsworth et al., 2012). This approach is being adopted in the following study.

\section{CONSTITUTIVE MODEL}

For the description of material stress-strain behaviour, it is typically assumed that:

$$
d \boldsymbol{\varepsilon}=d \boldsymbol{\varepsilon}_{e l}+d \boldsymbol{\varepsilon}_{p l}+d \boldsymbol{\varepsilon}_{c r}
$$

where $d \boldsymbol{\varepsilon}, d \boldsymbol{\varepsilon}_{e l}, d \boldsymbol{\varepsilon}_{p l}$ and $d \boldsymbol{\varepsilon}_{c r}$ are increments of total (mechanical), elastic, plastic and creep strain tensors. Based on Hook's law, the elastic contribution is:

$d \boldsymbol{\varepsilon}_{e l}=\mathbf{C}^{-1} \cdot d \boldsymbol{\sigma}$

where $d \boldsymbol{\sigma}$ is the increment of the stress tensor and $\mathbf{C}$ is the fourth order elasticity tensor. Hosseini et al (2015b) showed that, at least for the investigated $10 \% \mathrm{Cr}$ steel and for the midlife cycle condition, the elastic modulus is only a function of temperature and its variation for different heats of the alloy and for $\mathrm{LCF}$ and $\mathrm{C} / \mathrm{H}$ conditions is negligible. They described the temperature dependency of the elastic modulus for mid-life cycle condition as:

$$
E=x_{01} \cdot\left[1-x_{02} \exp \left(T / x_{03}\right)\right]
$$

where $x_{01-03}$ are material parameters and $T$ is the absolute temperature in Kelvin.

Determination of the plastic and creep incremental strain tensors requires definition of (cyclic) plasticity and creep constitutive models.

\section{Cyclic Plasticity}

The Mises yield locus for a strain hardening material can be written as: 
$f=\sqrt{\frac{3}{2}\left(\boldsymbol{\sigma}^{\prime}-\boldsymbol{\alpha}^{\prime}\right)\left(\boldsymbol{\sigma}^{\prime}-\boldsymbol{\alpha}^{\prime}\right)}-\sigma_{Y}$

where $\boldsymbol{\sigma}^{\prime}, \boldsymbol{\alpha}^{\prime}$ and $\sigma_{\mathrm{Y}}$ are deviatoric stress tensor, deviatoric backstress tensor and yield stress, respectively. Variations of $\boldsymbol{\alpha}$ and $\sigma_{\mathrm{Y}}$ with strain accumulation introduce kinematic and isotropic hardening to the yield locus. Isotropic hardening is usually included to represent the transition between virgin and mid-life cycle material conditions. For representation of the material deformation response at the mid-life cycle, isotropic hardening is usually neglected and $\sigma_{\mathrm{Y}}$ is only a function of temperature, i.e. $\sigma_{\mathrm{Y}}=\sigma_{\mathrm{Y}}(T)$. For a kinematic hardening material under deformation, the yield locus shifts in the stress space. Description of this shift can be defined by introducing a backstress tensor and a hardening rule which defines evolution of the backstress tensor by plastic strain accumulation. One of the best-known kinematic hardening models was proposed by Chaboche (1986):

$\boldsymbol{\alpha}=\sum_{i=1}^{m} \boldsymbol{\alpha}_{i}$

$d \boldsymbol{\alpha}_{i}=\frac{2}{3} C_{i} d \boldsymbol{\varepsilon}_{p l}-\gamma_{i} \boldsymbol{\alpha}_{i}\left|d \boldsymbol{\varepsilon}_{p l}\right|+\frac{1}{C_{i}} \frac{\partial C_{i}}{\partial T} \boldsymbol{\alpha}_{i} d T$

where $C_{i}$ and $\gamma_{i}$ are material/temperature dependent constants and $m$ is the number of backstress terms which is often considered to be 3 ('three term backstress Chaboche model').

The dependency of the Chaboche model parameters on temperature has been discussed by Hosseini et al. (2015b). They showed that an exponential equation-form can be used to describe the variation of model parameters with temperature. Hosseini et al. (2015a) also noted that, at least for the investigated $10 \% \mathrm{Cr}$ steel, irrespective of temperature, the yield strength $\left(\sigma_{\mathrm{Y}}\right)$, rate of strain hardening $\left(C_{i}\right)$ and maximum amount of strain hardening $\left(C_{i} / \gamma_{i}\right)$ for each heat of the alloy is linearly proportional to the room temperature $0.2 \%$ proof strength (i.e. $R_{p 0.2, R T}$ ) of that specific heat of the alloy.

Post-test metallographic examination after LCF and $\mathrm{C} / \mathrm{H}$ testing reveals the development of different material microstructures due to the introduction of hold times at peak strain in tension (e.g. Fournier et al., 2009). Such changes due to interacting creep deformation should be reflected in the Chaboche model parameters. It is hence reasonable that the model parameters for LCF and $\mathrm{C} / \mathrm{H}$ conditions are systematically different. The difference is a result of interacting creep deformation and is expected to be negligible at low temperatures and to become significant at higher temperatures.

Employment of the above considerations led to the following Chaboche model parameter representation (Hosseini et al., 2015a):

$$
\begin{aligned}
& \sigma_{Y}=x_{04} \cdot\left[1-x_{05} \exp \left(T / x_{06}\right)\right] \cdot R_{p 0.2, R T} \cdot \eta_{\sigma_{Y}} \\
& C_{1-3}=x_{07-09} \cdot\left[1-x_{10} \exp \left(T / x_{11}\right)\right] \cdot R_{p 0.2, R T} \cdot \eta_{C} \\
& \gamma_{1-3}=x_{12-14} \cdot\left[1-x_{15} \exp \left(T / x_{16}\right)\right]
\end{aligned}
$$

For the LCF condition, $\eta_{\sigma_{\mathrm{Y}}}$ and $\eta_{C}$ are unity. For representative loading 'histories' in real components which are more similar to the $\mathrm{C} / \mathrm{H}$ condition:

$$
\begin{aligned}
& \eta_{\sigma_{Y}}=\left[1-x_{17} \exp \left(T / x_{18}\right)\right] /\left(1-x_{17}\right) \\
& \eta_{C}=\left[1-x_{19} \exp \left(T / x_{20}\right)\right] /\left(1-x_{19}\right)
\end{aligned}
$$

Parameters $\eta_{\sigma_{\mathrm{Y}}}$ and $\eta_{C}$ are close to unity for low temperatures (lower than the insignificant creep temperature) but rapidly decrease at temperatures close to the maximum application temperature of the steel. 

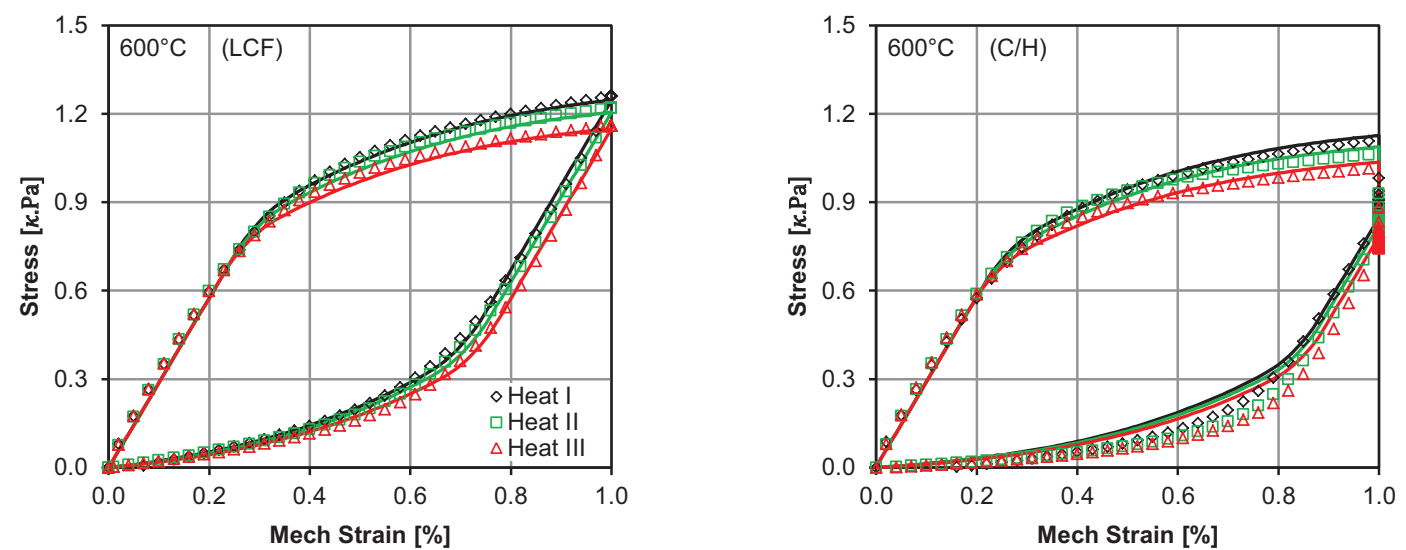

Figure 1. Comparisons of simulated (lines) and experimental (symbols) mid-life cycle stress-strain responses for different heats of the $10 \% \mathrm{Cr}$ steel under LCF and $\mathrm{C} / \mathrm{H}$ loading conditions at $600^{\circ} \mathrm{C}$

The effectiveness of the above model for representing the $10 \% \mathrm{Cr}$ steel cyclic deformation response have been reported by Hosseini et al. (2015a,b). As an example, Figure 1 compares the model representation for three heats of the investigated $10 \% \mathrm{Cr}$ steel under $\mathrm{LCF}$ and $\mathrm{C} / \mathrm{H}$ loading conditions at $600^{\circ} \mathrm{C}$.

The design assessment of actual components and structures is normally based on mean alloy deformation property representations. The mathematical representation introduced in this study enables determination of the Chaboche model parameters for different heats of the alloy based on their $R_{p 0.2, R T}$ values. Moreover, it is possible to define scatter band and mean value for the alloy cyclic response parameters on the basis of scatter band and mean $R_{p 0.2, R T}$ values (typically known from the alloy specification and/or data sheet).

\section{Creep}

A stress-regime and temperature dependent creep constitutive model has recently been introduced by Hosseini et al. (2015c). The model, which is a refinement of the 'Characteristic Strain' model developed by Bolton (2008), presents the isochronous stress-creep strain response of a material as:

$\varepsilon_{c}=\frac{0.2 \%\left(R_{R / t / T} / R_{0.2 / t / T}\right)}{R_{R / t / T} / \sigma-1}$

where $R_{R / t / T}$ and $R_{0.2 / t / T}$ are the rupture and $0.2 \%$ creep strengths of the material at the corresponding time and temperature. Definition of the dependency of $R_{R / t / T}$ and $R_{0.2 / t / T}$ on time and temperature extends this formula to variant temperature and time conditions. Assuming the Minimum Commitment (MC) relationship, developed by Manson and Ensign (1978), gives:

$$
\ln t=\beta_{0}+\beta_{1} \ln R_{R / t / T}+\beta_{2} R_{R / t / T}+\beta_{3} R_{R / t / T}^{2}+\beta_{4} T+\beta_{5} / T
$$

where $\beta_{0-5}$ are numerical constants. Equation (13) may then be solved to determine $R_{R / t / T}$ as a function of temperature and time.

A review of creep data for a number of power plant steels (High Temperature Design Data for Ferritic Pressure Vessel Steels, 1983) indicates that the creep strength of a material (e.g. $R_{0.2 / t / T)}$ is related to the rupture strength, e.g.

$$
R_{0.2 / t / T}=a_{1} R_{R / t / T}+a_{2} R_{R / t / T}^{2}+a_{3} R_{R / t / T}^{3}
$$

where $a_{1-3}$ are numerical constants. The simultaneous solution of equations (12-14) gives a stress-regime and temperature dependent creep constitutive model. The above model has been further refined in this 
study to represent the heat-to-heat variability and the effect of cyclic plasticity on the material creep deformation response.

For cyclic softening materials, the creep deformation resistance decreases as a consequence of the accumulation of inelastic strain. The effect of cyclic plasticity on the creep deformation response, determined by the above creep model, has been accomodated by introducing the parameter $Z_{C}$ in equation (13):

$$
\ln t=\beta_{0}+\beta_{1} \ln \left(\frac{R_{R / t / T}}{Z_{c}}\right)+\beta_{2}\left(\frac{R_{R / t / T}}{Z_{c}}\right)+\beta_{3}\left(\frac{R_{R / t / T}}{Z_{c}}\right)^{2}+\beta_{4} T+\beta_{5} / T
$$

where $Z_{C}$ is unity for the virgin material-condition. Parameter $Z_{C}$ for the mid-life cycle condition can be iteratively determined by comparison of the first and mid-life cycle relaxation behaviour of the alloy. Comparison of the determined $Z_{C}$ values for temperatures of 550 and $600{ }^{\circ} \mathrm{C}$ for the investigated $10 \% \mathrm{Cr}$ steel indicates that the $Z_{C}$ parameter decreases with increase of temperature. The variation can be described as:

$$
Z_{c}=\left[1-\chi_{1} \exp \left(T / \chi_{2}\right)\right] /\left(1-\chi_{1}\right)
$$

where $\chi_{1-2}$ are numerical constants. Parameters $Z_{C}$ is close to unity for low temperatures (lower than the insignificant creep temperature) but rapidly decrease at temperatures close to the maximum application temperature of the steel.

The influence of heat-to-heat variability on creep deformation resistance of the alloy may conceptually be covered by introducing term $Z_{s}$ into Equation (15), where $Z_{s}$ is unity and 0.8 for mean and minimum alloy property representations, respectively:

$$
\ln t=\beta_{0}+\beta_{1} \ln \left(\frac{R_{R / t \mid T}}{Z_{c} Z_{s}}\right)+\beta_{2}\left(\frac{R_{R / t / T}}{Z_{c} Z_{s}}\right)+\beta_{3}\left(\frac{R_{R / t \mid T}}{Z_{c} Z_{s}}\right)^{2}+\beta_{4} T+\beta_{5} / T
$$

For individual heats of the alloy, parameter $Z_{s}$ can be determined by comparison of the individual heat creep rupture/deformation behaviour with the alloy mean representation. The above model is relatively simple to derive and implement and is relevant for applications involving combinations of large and small stresses at various temperatures for virgin and midlife cycle conditions and for mean and minimum alloy property representations. Figure 2 demonstrates the effectiveness of the creep model for representing the constant load creep and stress relaxation behaviour of the $10 \% \mathrm{Cr}$ steels. Implementation of the derived creep model into ABAQUS commercial finite element (FE) code used the "CREEP" subroutine.
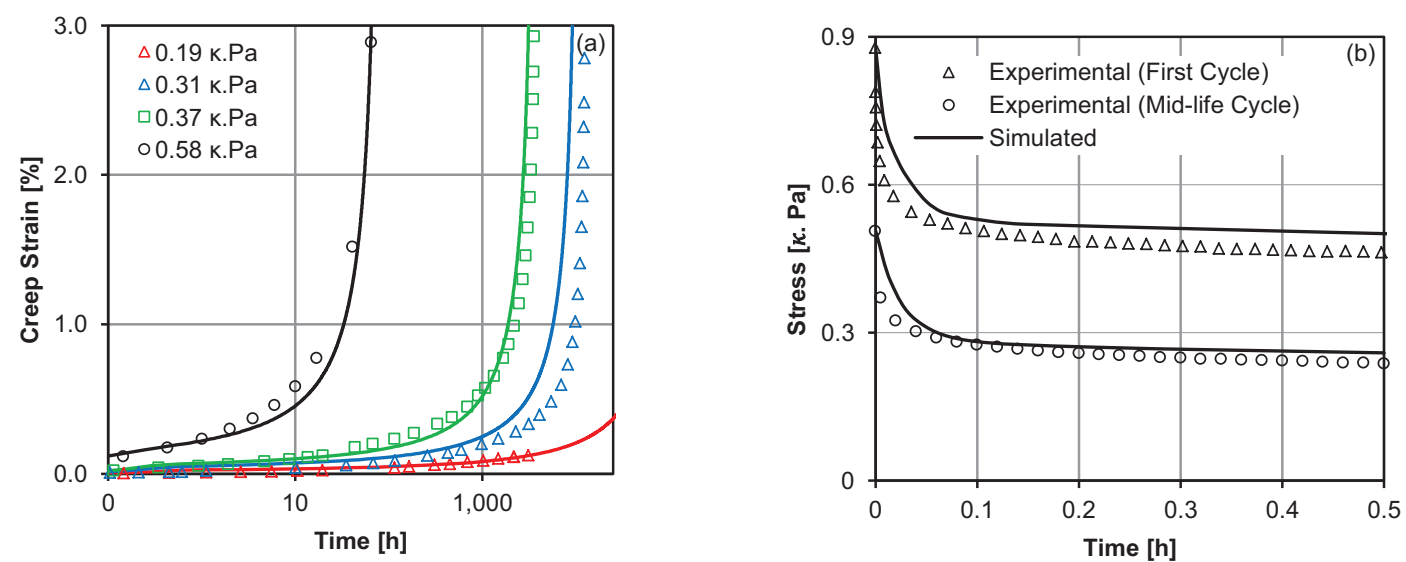

Figure 2. Comparisons of the creep model representation (lines) with available experimental data (symbols) under (a) constant load creep (virgin) and (b) stress relaxation (first and midlife cycles) testing conditions for $10 \% \mathrm{Cr}$ steel at $600^{\circ} \mathrm{C}$ 
An important reported observation from creep under cyclic loading conditions of some alloys is that for a crept material, inelastic straining in different/opposite directions clears the pervious strain-hardening memory. This means that for a crept sample to the secondary creep stage, a change of deformation direction follows by an enhanced creep rate, due to occurrence of primary creep persistency (PCP). Experimental evaluations showed that for the investigated $10 \% \mathrm{Cr}$ steel, stress/deformation reversal involving a minimum of $0.01 \%$ inelastic strain accumulation of the opposite sign, to that last accumulated, fully clears the pervious strain-hardening memory. The developed "CREEP" subroutine for the derived creep constitutive model uses a strain hardening assumption and clears the pre-strain history after a change in the sign of the sum of maximum and minimum principal stresses, provided that a minimum of $0.01 \%$ inelastic strain had been accumulated since the last stress/deformation reversal.

\section{CREEP-FATIGUE DAMAGE}

The following describes the formulation employed in this creep-fatigue assessment procedure for calculation of the creep and fatigue damage fractions. A life usage factor (LUF) parameter has then been introduced which accounts for the accumulation of individual creep and fatigue damage fractions as well as their possible interaction.

\section{Fatigue Damage}

Consistent with ASME III, RCC-MR and R5, fatigue damage fraction may be calculated as:

$d_{F}^{*}=\frac{1}{N_{i}}$

where $d_{F}^{*}$ is the generated fatigue damage fraction per cycle and $N_{i}=N_{i / \Delta \varepsilon / T}$ is the reference crack initiation endurance which is inherently a function of temperature and strain range (and material). The present creep-fatigue assessment procedure considers:

$$
d_{F}^{*}=\frac{1}{N_{i / \Delta \varepsilon^{*} / T_{\max }}}
$$

where the reference crack initiation endurance refers to the minimum isothermal LCF (without hold time) endurance. Assessment of components by means of FE analysis enables calculation of the stress and strain vectors in different directions for individual elements. Parameter $\Delta \varepsilon^{*}$ in above equation is the FE calculated total (mechanical) strain range at the element centroid in the direction which gives the maximum strain range (this direction is most often parallel to the component surface). Parameter $T_{\max }$ in equation (19) is the maximum temperature of the element during the operating cycle.

\section{Creep Damage}

The present creep-fatigue assessment procedure estimates the developed creep damage based on the ductility exhaustion approach, adopted by Hales (1983):

$$
d_{C}^{*}=\int_{0}^{t_{h}}\left[\dot{\varepsilon}_{\mathrm{cr}}^{*}(t) / \varepsilon_{\mathrm{R}}\left(\dot{\varepsilon}_{\mathrm{c}}^{*}\right)\right] \cdot d t
$$

where $d_{C}^{*}$ is the creep damage fraction accumulated per cycle, $t_{h}$ is the dwell (steady operation) time per cycle and $\varepsilon^{*}$ cr is the FE calculated creep strain rate at the element centroid in the direction which gives the maximum total (mechanical) strain range. Physical creep damage is assumed not to occur as a consequence of compressive strain accumulation, i.e. when $\varepsilon^{*}{ }_{\text {cr }}^{*}<0$. Parameter $\varepsilon_{R}$ is the alloy minimum creep ductility expressed in terms of true strain at rupture as a function of true creep strain rate. 


\section{Life Usage Factor}

The concept of creep-fatigue assessment consists in determination of the capacity of a component to sustain a number of well-defined cyclic deformation periods. In other words, a creep-fatigue assessment should determine the life usage factor (LUF) for critical locations of the component after the defined number of thermomechanical loading cycles. The outcome of an FE analysis of the component is used to define the characteristics of the thermomechanical cycle for individual FE mesh elements and equations (19) and (20) can then provide the fatigue and creep damage fractions at critical locations in the component. An empirical damage summation locus may finally be used for determination of the LUF based on consideration of creep and fatigue damage fractions, as well as their possible interactions. The damage summation locus is dependent not only on material, but also on the assessment procedure used to determine individual fatigue and creep damage fractions.

A linear damage summation rule may be used for alloys with insignificant creep-fatigue damage interaction (Figure 3). The governing equation for such a creep-fatigue interaction locus is:

$D_{F}^{C F I}=1-D_{C}^{C F I}$

where $D^{C F I}$ and $D_{F}^{C F I}$ are creep and fatigue damage fraction coordinates on the interaction locus. Other damage summation rules may be used for alloys with higher creep-fatigue damage interaction characteristics. For example for $9 \mathrm{Cr}-1 \mathrm{Mo}-\mathrm{V}$ steel, ASME III proposed:

$$
\begin{array}{ll}
D_{F}^{C F I}=1-90 \cdot D_{C}^{C F I} & \text { for } D_{C}^{C F I}<0.01 \\
D_{F}^{C F I}=\left(1-D_{C}^{C F I}\right) / 9.9 & \text { for } D_{F}^{C F I}>0.01
\end{array}
$$

Observation on the investigated $10 \% \mathrm{Cr}$ steel under creep-fatigue loading conditions revealed a high level of creep-fatigue interactions. A creep-fatigue interaction locus was then proposed as:

$$
D_{F}^{C F I}=\left[1-\left(D_{C}^{C F I}\right)^{0.15}\right]^{0.75}
$$

For cumulated creep and fatigue damage fractions of $D_{C}^{*}=\Sigma d_{C}^{*}$ and $D_{F}^{*}=\Sigma d_{F}^{*}$, the LUF factor is:

$$
\mathrm{LUF}=\left\{\left[\left(D_{\mathrm{F}}^{*}\right)^{2}+\left(D_{\mathrm{C}}^{*}\right)^{2}\right] /\left[\left(D_{\mathrm{F}}^{\mathrm{CFI}}\right)^{2}+\left(D_{\mathrm{C}}^{\mathrm{CFI}}\right)^{2}\right]\right\}^{0.5}
$$

with $D_{F}^{C F I}\left(D_{C}^{C F I}\right)$ defined by equation (23). The LUF for a safe design should be less than unity for all locations of the structure/component.

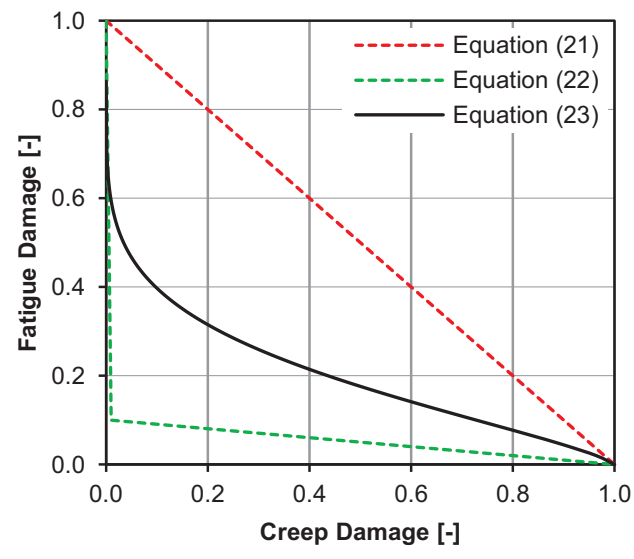

Figure 3. Representation of creep-fatigue damage loci by equations (21-23)

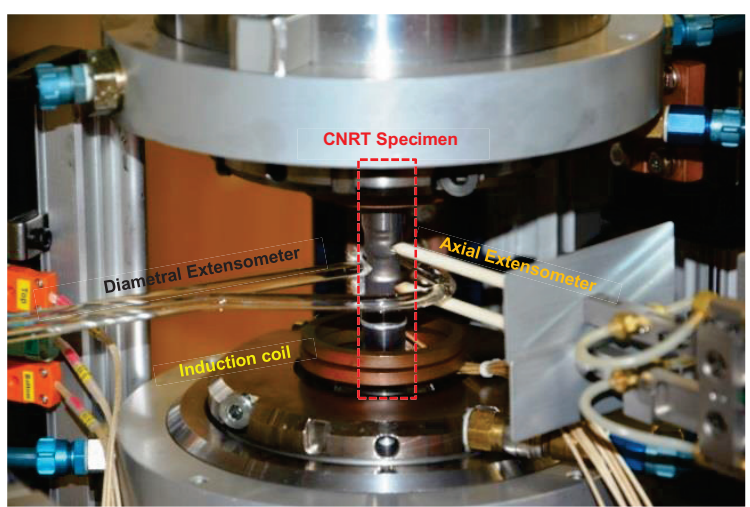

Figure 4. The test setup used for the benchmark testing 


\section{EFFECTIVE VERIFICATION}

Verification of the effectiveness of complete creep-fatigue assessment procedures is typically based on the results of service experience. A solution in the absence of appropriate service experience is to employ component-feature specimen service-cycle TMF tests. Component feature specimen service-cycle TMF benchmark test design involves the following steps (Holdworth et al. 2012):

i) Thermo-mechanical analyses of a typical component to identify the creep-fatigue life limiting feature location and operating cycle

ii) Determination of the cyclic stress-strain hysteresis behaviour at the life limiting location, and the associated stress/strain states (at, and below the surface) in terms of

- Mechanical strain range,

- Stress triaxiality factor (i.e. ratio of maximum to Mises stresses), and

- Creep strain accumulated per cycle

iii) Design of a laboratory cycle to replicate, as closely as possible, the component-feature in-service stress-strain hysteresis behaviour, and

iv) Design of a laboratory specimen with a geometrical feature having the stress/strain characteristics at (and below) the groove root surface to be as close as possible to those defined in ii)

FE simulations of a component of interest during a typical start-up condition were first performed, using the constitutive model presented in this study for mean $10 \% \mathrm{Cr}$ steel properties, to establish the stress/strain state at the life limiting feature location. Predictably, this assessment revealed a critical location which in a service-cycle test with representative start-up (loading), hold and shut-down (unloading) durations would result in an impractically long laboratory test time. TMF benchmark tests are typically designed to last for around 4 to 6 weeks. In order to reduce the expected component lifetime, start-up, hold and shutdown times were reduced in the component simulations with the objective of generating enhanced service representative stress/strain state distributions to form the basis of componentfeature specimen design. Based on the results of FE analysis of the enhanced start-up conditions for the actual component, the optimised geometry and TMF cycle shape for the benchmark test were designed. The optimised geometry is a circumferentially notched round tensile (CNRT) specimen comprising a notch with radius and depth of $12.5 \mathrm{~mm}$ and $1.0 \mathrm{~mm}$, respectively. The specimen had a notch root diameter of $12 \mathrm{~mm}$ (see Figure 4).

Figure 5a shows the FE calculated temperature cycles for the life limiting feature locations of the actual component (as a dotted line) and the CNRT specimen (as a solid line). The mechanical strain cycle at the life limiting location of the actual component is shown in Figure 5b (dotted line). A remote axial mechanical displacement cycle was designed in a way that its application to the central $15 \mathrm{~mm}$ axial length of the CNRT specimen generated the notch root mechanical strain cycle shown in Figure 5b (solid line). An example of a stabilised stress-strain loop associated with the enhanced start-up conditions for the actual component life limiting feature is shown as the dotted hysteresis loop in Figure 5c. The consequent stress/strain loop at the notch root of the CNRT specimen is shown as a solid line in Figure 5c. Figure 6 shows verification of the physical reality of the designed benchmark test based on comparisons of the gradients of strain range (a), stress multi-axiality factor (b) and cumulated creep strain per cycle (c), in the direction perpendicular to the surface for the CNRT specimen notch root with respect to the life limiting feature location of the actual component.

Component feature specimen service-cycle TMF benchmark tests have been designed to check the effectiveness of the introduced creep-fatigue assessment procedure. While the applied temperature cycle for all the tests is similar to the temperature cycle introduced in Figure 5a, the mechanical displacement cycle varies. Figure 4 shows the developed testing setup for the component feature specimen servicecycle TMF benchmark tests. 

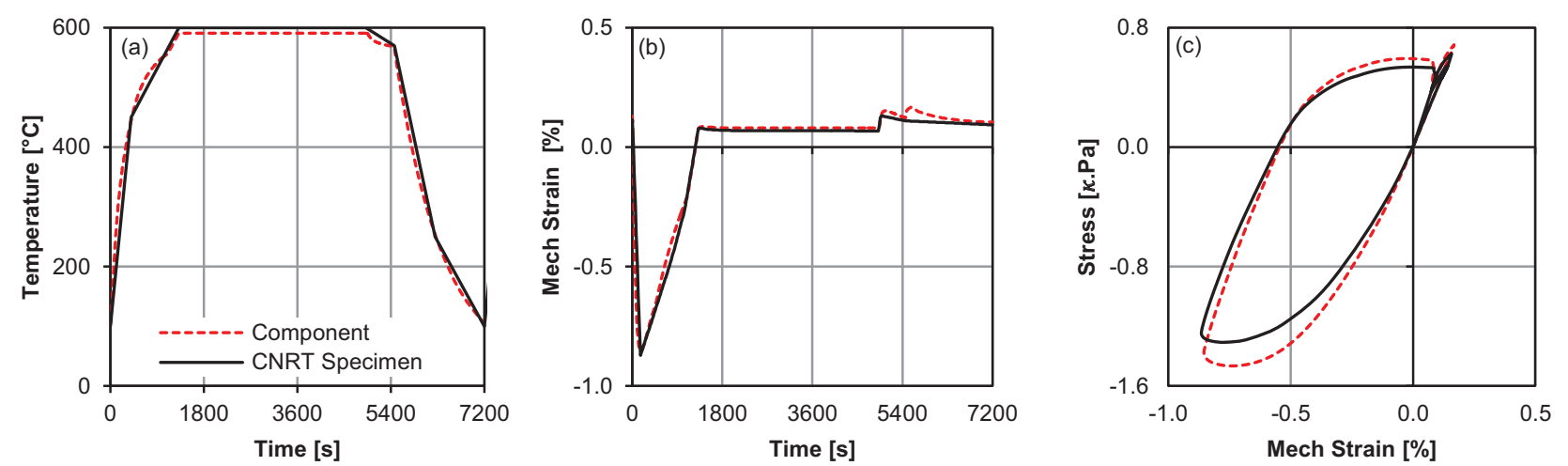

Figure 5. Comparisons of FE calculated (a) temperature cycles, (b) mechanical strain cycles and (c) hysteresis loops for groove root of CNRT testpiece subjected to the service-type TMF cycle and for actual component life limiting feature during the enhanced start-up condition
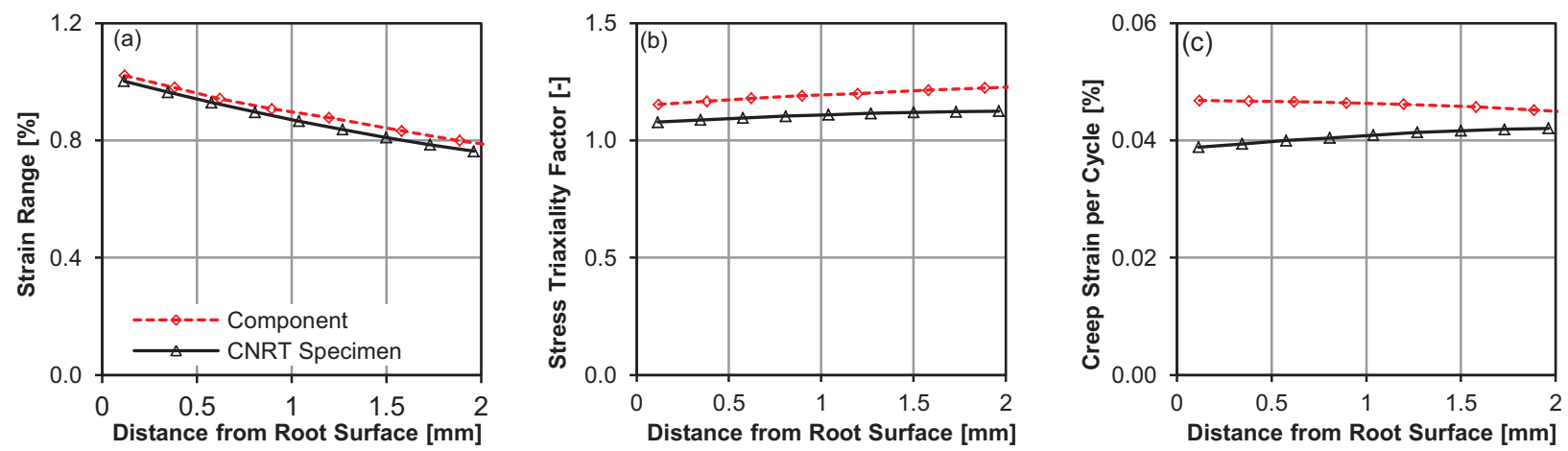

Figure 6. Comparisons of stress/strain distribution criteria at groove root of CNRT testpieces subject to service-type TMF cycle with that at the component life limiting feature during enhanced start-up conditions: variation with distance from groove root of (a) mechanical strain range, (b) stress triaxiality factor, and (c) creep strain accumulated per cycle

\section{CONCLUDING REMARKS}

This study introduces a creep-fatigue assessment procedure for calculating the life time of high temperature components of power plant (e.g. rotating turbo-machinery parts) under fast start-up and shutdown conditions. The procedure considers alloys with high creep-fatigue deformation/damage interaction characteristics such as the advanced martensitic $9-11 \% \mathrm{Cr}$ steels which are widely used for power plant applications. The procedure takes advantages of advanced constitutive models for both cyclic plasticity and creep and implements them in a state-of-the-art mechanical assessment procedure for calculating high temperature component life times. The employed cyclic plasticity and creep models are temperature dependent developments of Chaboche kinematic hardening cyclic plasticity and 'Characteristic Strain' creep models which account for the mutual interaction of cyclic plasticity and creep deformation. Design analysis of real components cannot be based on the behaviour description of a single heat of an alloy and is typically based on mean alloy properties for the determination of creep-fatigue deformation, and minimum alloy properties for the determination of creep-fatigue damage behaviour. The cyclic plasticity and creep constitutive models are therefore refined to represent the heat-to-heat variability of the alloy deformation response to represent the mean properties. Application of the constitutive models in finite element analysis of the high temperature component determines the stress-strain state at life limiting features locations. Such stress-strain information can then be employed by conventional analytical formulations to calculate the developed creep and fatigue damage fractions and their possible interaction 
to eventually evaluate the safety of the designed structure/component. In the absence of appropriate service experience for power plant components manufactured from $9-11 \% \mathrm{Cr}$ steel and operated in a more flexible way, the effectiveness of the assessment procedure is being verified using results from a number of component feature specimen service-cycle thermomechanical fatigue benchmark tests.

\section{REFERENCES}

ASME III. ASME Boiler and Pressure Vessel Code, Section III, Division 1: Sub-section NH, Class 1 Components in Elevated Temperature Service, New York, USA.

Bolton, J. (2008). “A 'Characteristic-Strain' model for creep," Materials at High Temperatures, Vol 25(3), 197-204.

Chaboche, J. L. (1986). "Time-independent constitutive theories for cyclic plasticity," International Journal of Plasticity, Vol 2(2), 149-188.

Fournier, B., Sauzay, M., Caës C., Mottot, M., Noblecourt, M. and Pineau, A. (2009). "Creep-fatigue interactions in a 9Cr-1Mo martensitic steel: part II. microstructural evolutions," Metallurgical and Materials Transactions A, Vol 40(2), 330-341.

Hales, R. (1983). "A method of creep damage summation based on accumulated strain for the assessment of creep-fatigue endurance," Fatigue \& Fracture of Engineering Materials \& Structures, Vol 6(2), 121-135.

High Temperature Design Data for Ferritic Pressure Vessel Steels, (1983). Institution of Mechanical Engineers (Great Britain). Creep of Steels Working Party, Mechanical Engineering Publications.

Holdsworth, S. R., Radosavljevic, M., Grossmann, P., Kühn, I. and Mazza, E. (2012). "Effectiveness verification of creep-fatigue assessment procedures for fast-starting steam turbines," in $A S M E$ Turbine Technical Conference and Exposition, American Society of Mechanical Engineers, 367373.

Hosseini, E., Holdsworth, S. R., Kühn, I. and Mazza, E. (2015a). "Modelling heat-to-heat variability in high temperature cyclic deformation behaviour," Materials at High Temperatures, Vol 32(3), 347354.

Hosseini, E., Holdsworth, S. R., Kühn, I. and Mazza, E. (2015b). "Temperature dependent representation for Chaboche kinematic hardening model," Materials at High Temperatures, Vol 32(4), 404-411.

Hosseini, E., Holdsworth, S. R. and Mazza, E. (2015c). "Temperature and stress-regime dependent primary-secondary-tertiary creep constitutive model," Materials at High Temperatures, Vol 32(4), 384-389.

Kachanov, L. (2004). Fundamentals of the theory of plasticity, Courier Dover Publications. Mineola, New York, USA.

Manson, S. and Ensign, C. (1978). "Interpolation and extrapolation of creep rupture data by the minimum commitment method," in Characterization of materials for service at elevated temperatures, MPC7, ASME, New York, USA, 299-398.

R5. Assessment Procedure for the High Temperature Response of Structures, British Energy, Gloucester, UK.

Radosavljevic, M. (2011). "Creep-fatigue assessment of high temperature steam turbine rotors (Diss ETH No. 19880)," PhD thesis, ETHZ University, Zürich, Switzerland.

RCC-MR. Design and Construction Rules for Mechanical Components of FBR Nuclear Islands, AFCEN, Paris, France. 